\title{
Relation between type of hysterectomy and survival rate in patients with ovarian cancer - single-center study
}

\author{
Stanislav Slavchev ${ }^{1}$, Stoyan Kostov ${ }^{1}$, Strahil Strashilov ${ }^{2}$, Angel Yordanov \\ ${ }^{1}$ Department of Gynecology, Medical University of Varna, Bulgaria \\ ${ }^{2}$ Department of Plastic Restorative, Reconstructive and Aesthetic Surgery, Medical University of Pleven, Bulgaria \\ ${ }^{3}$ Department of Gynaecological Oncology, Medical University of Pleven, Bulgaria
}

\begin{abstract}
Aim of the study: To evaluate the survival rate of patients with advanced ovarian carcinoma in relation to the type of surgical intervention - total abdominal hysterectomy with bilateral adnexectomy and omentectomy as a minimal standard compared to extended hysterectomy with a retroperitoneal approach.

Material and methods: The study was implemented based on retrospectively obtained data from 104 patients operated on for advanced epithelial ovarian carcinoma (FIGO stages II-IV) in the period from 2004 to 2012. Total abdominal hysterectomy, bilateral adnexectomy, and omentectomy were performed on 23 patients. Extended hysterectomy with a retroperitoneal approach and varying degrees of peritonectomy, omentectomy, and appendectomy were performed on 74 patients. Seven patients were treated with adnexectomy or biopsy alone. We divided the patients into two groups according to the mode of surgery. The first one comprised the patients who underwent radical hysterectomy and the second one comprised total abdominal hysterectomy plus bilateral adnexectomy. The two groups were examined for their overall survival rate, relapse-free survival rate, and 5-year survival rate.

Results: Mean overall survival rate, relapse-free survival rate, and 5-year survival rate in the group with extended hysterectomy were higher compared to the group with total abdominal hysterectomy.

Conclusions: The extended hysterectomy with a retroperitoneal approach with or without systematic lymph node dissection seems to be more appropriate in the surgical treatment of advanced ovarian carcinoma. The procedure is related to the improvement of survival rate as a result of the inclusion of macroscopically invisible lesions in the surgical removal.
\end{abstract}

Key words: ovarian carcinoma, radical hysterectomy, overall survival, 5-year survival rate.

\section{Introduction}

The EUROCARE-5 population study based on 107 oncological registers in 29 European countries for the period from 1999 to 2007 reported a 37.6\% 5-year survival rate of ovarian cancer in all stages of the disease [1]. According to the CONCORD-2 program based on cancer registers in 61 countries, the 5-year survival rate for ovarian carcinoma in the advanced stage was $30 \%$, and it was significantly lower compared to the disease limited to the pelvis, in which case the survival rate reached $80 \%$ [2]. Advanced ovarian carcinoma (AOC) remains a challenge for surgeons due to the need for complete surgical extirpation of the tumor with the purpose of extension of the period of survival. The standard surgical intervention, according to the guidelines, remains the total abdominal hysterectomy (TAH) with bilateral salpingo-oophorectomy and omentectomy [3]. Radical pelvic surgery, including hysterectomy, became necessary for a significant proportion of patients. A surgical technique was described in 1968 by Hudson and Chir, in which the retroperitoneal approach to the pelvic structures allows the complete removal of the neoplasm, without it being resected and without the persistence of residual tumor [4]. The procedure includes en bloc extirpation of the uterus with both adnexa, pelvic peritoneum with or without resection of the rectosigmoid colon and partial peritonectomy. The surgical technique for the retroperitoneal approach is similar to that for radical hysterectomy for cervical cancer. This type of hysterectomy can be termed extended hysterectomy (EH). Our study aims to assess the significance of $\mathrm{EH}$ for the overall and relapse-free survival rate.

\section{Material and methods}

The data were collected retrospectively from the hospital records of 104 patients operated on in the 
period from 2004 to 2012 at the Clinic of Gynecology of General Hospital for Active Treatment „Saint Anna” - Varna City for the treatment of AOC (FIGO stages IIIV). The patients were analyzed with respect to the age, FIGO stage, histological variant and grading of tumor, size and location of the residual tumor, and types of hysterectomy - TAH, EH, and other surgical procedures. The staging was implemented according to the FIGO classification from 1998. 95\% of patients received adjuvant chemotherapy in six cycles of combined chemotherapy with carboplatin (in a dose calculated by area under concentration-time curve [AUC] of 5-7.5) and paclitaxel of $175 \mathrm{mg} / \mathrm{m}^{2}$ administered once every three weeks or with an alternative regimen - combination of paclitaxel of $135 \mathrm{mg} / \mathrm{m}^{2}$ on day 1 and $60 \mathrm{mg} / \mathrm{m}^{2}$ on

Table 1. Characteristics of the population of patients

\begin{tabular}{|c|c|c|}
\hline Characteristics & $n$ & $\%$ \\
\hline \multicolumn{3}{|l|}{ Average age $60.84 \pm 11.67$} \\
\hline \multicolumn{3}{|l|}{ FIGO stage } \\
\hline$\| \mathrm{A}$ & 4 & 3.8 \\
\hline IIB & 4 & 3.8 \\
\hline IIC & 5 & 4.8 \\
\hline IIIA & 3 & 2.9 \\
\hline IIIB & 11 & 10.6 \\
\hline IIIC & 63 & 60.6 \\
\hline IV & 14 & 13.5 \\
\hline \multicolumn{3}{|l|}{ Histological variant of tumor } \\
\hline Serous & 88 & 84.6 \\
\hline Non-differentiated & 8 & 7.7 \\
\hline Mucinous & 5 & 4.8 \\
\hline Clear cell & 2 & 1.9 \\
\hline Endometrioid & 1 & 1 \\
\hline \multicolumn{3}{|l|}{ Grade of differentiation of tumor } \\
\hline G1 & 4 & 3.8 \\
\hline $\mathrm{G} 2$ & 67 & 64.4 \\
\hline G3 & 33 & 31.7 \\
\hline \multicolumn{3}{|l|}{ Ascites } \\
\hline No ascites & 38 & 36.5 \\
\hline Ascites (positive for tumor cells) & 43 & 41.3 \\
\hline Ascites (negative for tumor cells) & 23 & 22.1 \\
\hline \multicolumn{3}{|l|}{ Location of the primary tumor } \\
\hline Right ovary & 32 & 30.8 \\
\hline Left ovary & 20 & 19.2 \\
\hline Both ovaries & 50 & 48.1 \\
\hline \multicolumn{3}{|l|}{ Concomitant diseases } \\
\hline CVD & 51 & 49 \\
\hline DM & 13 & 12.5 \\
\hline$C V D+D M$ & 8 & 7.7 \\
\hline
\end{tabular}

day 8 with cisplatin of $75-100 \mathrm{mg} / \mathrm{m}^{2}$ once every three weeks. Neoadjuvant chemotherapy was administered to a minority of patients with paclitaxel of $175 \mathrm{mg} / \mathrm{m}^{2}$ and carboplatin (AUC of 6-5). Patients were monitored during 60-120 months. Information about their live status was obtained from the National cancer register.

The data from the study were entered and analyzed with the statistical software MedCalc2018. Descriptive analysis was applied for the determination of an average value, standard deviation, and median. The curves of survival rate were drawn by the method of Kaplan-Meier and were estimated with the log-rank test. The factors and differences with the level of significance of $<0.05(p<0.05)$ in all analyses were considered statistically significant.

\begin{tabular}{|c|c|c|}
\hline Characteristics & $n$ & $\%$ \\
\hline Primary multiplicity & 11 & 10.6 \\
\hline Others & 8 & 7.7 \\
\hline No concomitant diseases & 27 & 26 \\
\hline \multicolumn{3}{|l|}{ Dimensions of surgical treatment } \\
\hline $\begin{array}{l}\text { TAH, bilateral adnexectomy, } \\
\text { omentectomy, appendectomy }\end{array}$ & 23 & 22.1 \\
\hline $\begin{array}{l}\text { EH with a retroperitoneal approach } \\
\text { with or without peritonectomy, } \\
\text { bilateral salpingo-oophorectomy }\end{array}$ & 74 & 71.2 \\
\hline $\begin{array}{l}\text { Other types of operations - } \\
\text { adnexectomy, biopsy, etc. }\end{array}$ & 7 & 6.7 \\
\hline \multicolumn{3}{|l|}{ Chemotherapy } \\
\hline Neoadjuvant & 3 & 2.9 \\
\hline Adjuvant & 89 & 85.6 \\
\hline Neoadjuvant + adjuvant & 8 & 7.7 \\
\hline Palliative & 2 & 1.9 \\
\hline No chemotherapy & 2 & 1.9 \\
\hline \multicolumn{3}{|l|}{ Residual tumor } \\
\hline $0-1 \mathrm{~cm}$ & 44 & 42.3 \\
\hline $1-2 \mathrm{~cm}$ & 20 & 19.2 \\
\hline more than $2 \mathrm{~cm}$ & 40 & 38.5 \\
\hline \multicolumn{3}{|l|}{ Location of residual tumor } \\
\hline No visible residual tumor & 26 & 25 \\
\hline Rectum, sigmoid colon & 43 & 41.3 \\
\hline Small intestines & 29 & 27.9 \\
\hline Liver & 17 & 16.3 \\
\hline Domes of the diaphragm & 28 & 26.9 \\
\hline Omentum & 14 & 13.4 \\
\hline Pleurae & 4 & 3.8 \\
\hline Urinary bladder & 3 & 2.9 \\
\hline Transverse colon & 2 & 1.9 \\
\hline
\end{tabular}

CVD - cardiovascular diseases, DM - diabetes mellitus, TAH - total abdominal hysterectomy, EH - extended hysterectomy 


\section{Results}

The characteristics of the population of patients are summarized in Table 1.

Among 23 patients (22.1\%) TAH with bilateral adnexectomy and omentectomy were performed. In 74 patients (71.2\%) EH with a retroperitoneal approach and some degree of peritonectomy with omentectomy were performed; only in six patients did the procedure include rectosigmoid resection. The two groups - with $\mathrm{TAH}$ and with $\mathrm{EH}$ - were juxtaposed and analyzed with respect to the overall survival and disease-free survival. Seven patients underwent palliative or diagnostic surgical procedures without eradication of the disease. The latter group was not included in the survival analysis. Table 2 shows comparative data for the group with $\mathrm{TAH}$ and the group with $\mathrm{EH}$.

The analysis of survival rate (Figs. 1 and 2), which covered patients of FIGO stages II-IV, did not demonstrate a statistically significant difference in the overall survival (OS), and disease-free survival (DFS) between the two groups: with TAH $(n=23)$ and $\mathrm{EH}(n=74)$ (48.4 months vs. 47.6 months for OS, and 27.7 months vs. 33.3 months for DFS).

Table 2. Comparison of groups with total and extended hysterectomy in relation to FIGO stage, residual tumor, grading, histological variant of tumor, and type of chemotherapy

\begin{tabular}{lcc}
\hline Parameter & $\begin{array}{c}\text { Total } \\
\text { hysterectomy } \\
n(\%)\end{array}$ & $\begin{array}{c}\text { Extended } \\
\text { hysterectomy } \\
n(\%)\end{array}$ \\
\hline Number of patients $(n=97)$ & 23 & 74 \\
\hline FIGO stage & & \\
\hline II $(n=13)$ & 8 & 5 \\
\hline III $(n=72)$ & 13 & 59 \\
\hline IV $(n=12)$ & 2 & 10 \\
\hline Residual tumor & & \\
\hline O-1 cm $(n=43)$ & 13 & 30 \\
\hline FIGO II & 6 & 3 \\
\hline FIGO III and IV & 7 & 27 \\
\hline$>1$ cm $(n=54)$ & 10 & 44 \\
\hline Grading & $1(4)$ & $3(4)$ \\
\hline G1 & $17(74)$ & $45(61)$ \\
\hline G2 & $5(22)$ & $26(35)$ \\
\hline G3 & $3(1.3)$ & $3(4)$ \\
\hline Histological variant & $22(96)$ & $73(95)$ \\
\hline Serous & $3(3.5)$ & $2(2.7)$ \\
\hline Mucinous & $0(0)$ & $1(1.4)$ \\
\hline Endometrioid & $0(0)$ & $6(2.7)$ \\
\hline Clear cell & & \\
\hline Non-differentiated & & \\
\hline Chemotherapy & $(85)$ \\
\hline Neoadjuvant & & \\
\hline Adjuvant & & \\
\hline & & \\
\hline
\end{tabular}

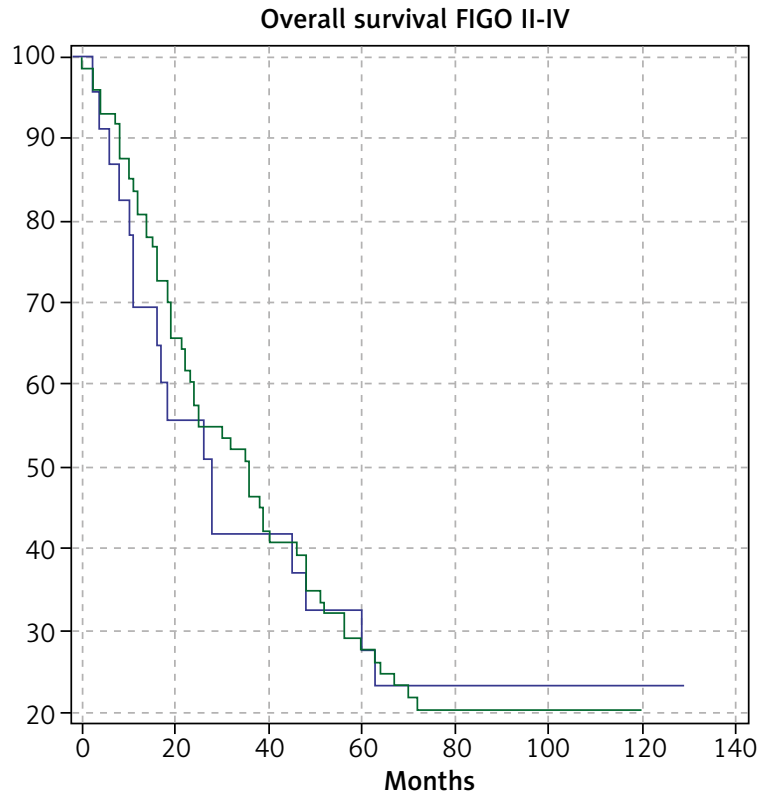

Types of hysterectomy

\begin{tabular}{|l|c|}
\multicolumn{2}{|c}{$-1-2$} \\
\hline$\chi^{2}$ & 0.03572 \\
\hline Degrees of freedom & 1 \\
\hline Significance & $p=0.8501$ \\
\hline
\end{tabular}

Fig. 1. Kaplan-Meier curve for overall survival of patients with ovarian carcinoma of FIGO stages II-IV, divided into two groups according to type of hysterectomy: group 1 with total abdominal hysterectomy (TAH), and group 2 with extended hysterectomy $(\mathrm{EH})$

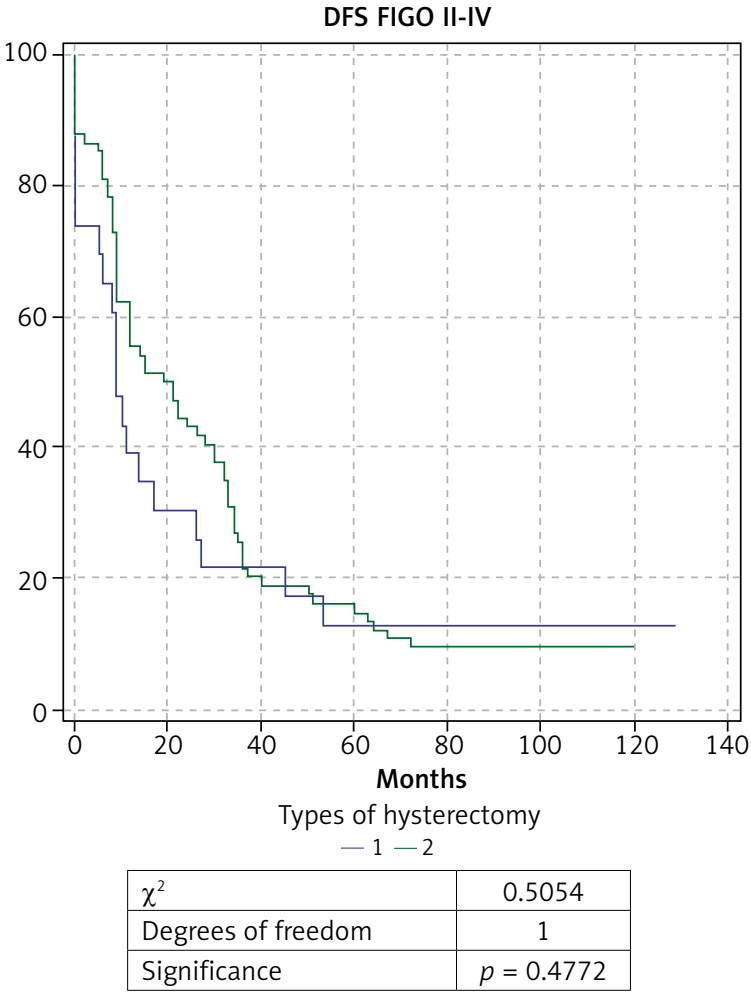

Fig. 2. Kaplan-Meier curve for disease-free survival (DFS) of patients with ovarian carcinoma of FIGO stages II-IV, divided into two groups according to type of hysterectomy: group 1 with total abdominal hysterectomy (TAH), and group 2 with extended hysterectomy $(\mathrm{EH})$ 


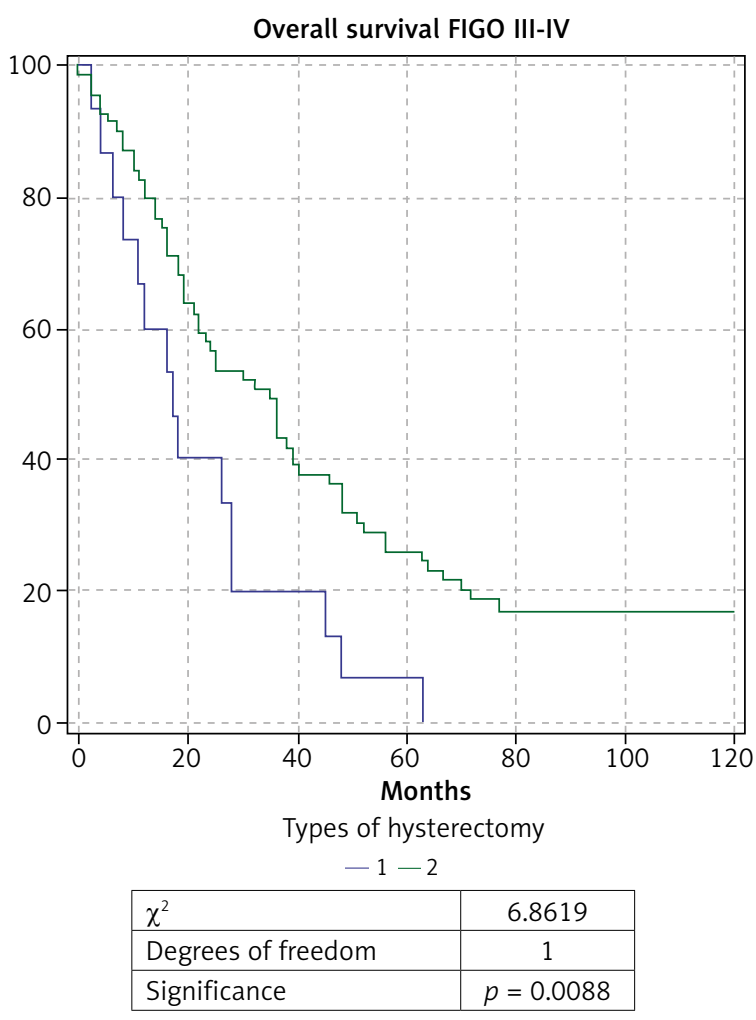

Fig. 3. Kaplan-Meier curve for overall survival rate of patients with ovarian carcinoma of FIGO stage III-IV, divided into two groups according to type of hysterectomy: group 1 with total abdominal hysterectomy (TAH), and group 2 with extended hysterectomy $(\mathrm{EH})$

When comparing the $\mathrm{EH}(n=69)$ and TAH $(n=15)$ in patients with AOC of stages III and IV a significantly higher OS rate, DFS rate, and 5-year survival rate were observed in the group of EH: respectively 44.7 months; 29 months and $26 \%$ compared to 22.1 months; 10.4 months and 7\% for TAH. The comparative Kaplan-Meier curves for the overall and disease-free survival rates for the subgroups with FIGO stages III-IV are shown in Figure 3 and Figure 4. We evaluated the survival rate in the subgroup of patients with FIGO III-IV and residual tumor of $0-1 \mathrm{~cm}$ to reduce the burden of the latter as a major predictor. A significant difference in the overall and relapse-free survival rate was also found in the subpopulation of patients with FIGO III-IV and an optimal cytoreduction (Figs. 5 and 6). The OS rate, DFS rate, and 5-year rate were respectively: 66.4 months; 41.3 months; $52 \%$ for $\mathrm{EH}(n=27)$ and 17.7 months; 8.4 months and $0 \%$ for TAH $(n=7)$.

\section{Discussion}

Optimal cytoreduction in the case of AOC can be achieved employing TAH with bilateral adnexectomy, and omentectomy. In our cohort, TAH was sufficient to achieve optimal cytoreduction for only 13 patients

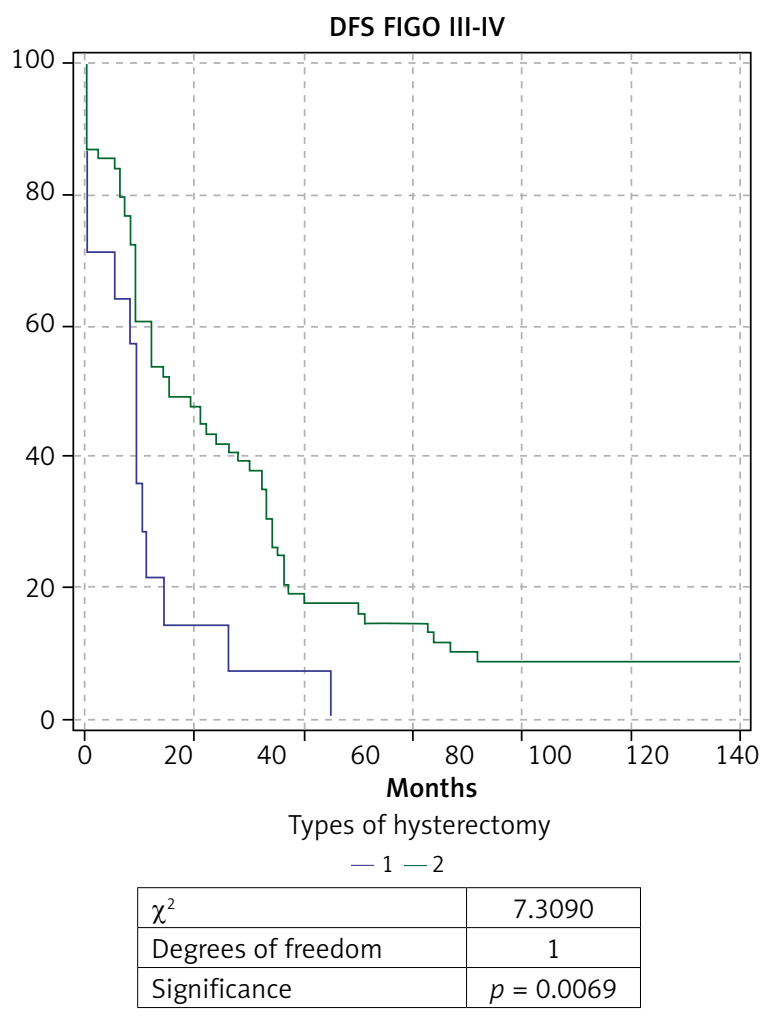

Fig. 4. Kaplan-Meier curve for disease-free survival (DFS) rate of patients with ovarian carcinoma of FIGO stage III-IV, divided into two groups according to type of hysterectomy: group 1 with total abdominal hysterectomy (TAH), and group 2 with extended hysterectomy (EH)

out of 104. Five-year survival was observed in only 6 of them, who were in FIGO stage II, and comprised about $50 \%$ of FIGO stage II of our sample. Ovarian carcinoma of FIGO stage II is hard to define and is found in about $10 \%$ of all cases [5]. Stage II, according to the FIGO classification of ovarian cancer, includes treatable tumors, tumors confined to the pelvis and metastatic tumors on the pelvic peritoneum, which usually have poorer prognosis [5]. Those facts show that the minimum standard requirement for surgical treatment of $\mathrm{AOC}$ would be sufficiently effective only for a small proportion of patients.

The $\mathrm{EH}$, on the other hand, contributes to improvement of overall and disease-free survival rate in patients in FIGO stage III and IV. Many authors have evaluated the $\mathrm{EH}$ and its modifications in ovarian carcinoma. However, not all of them succeeded in proving explicitly its benefit for the survival rate [6]. Alleti et al. [7] compared the radical pelvic surgery with rectosigmoid resection, or peritoneal stripping with standard pelvic surgery in patients with AOC of FIGO stages IIIC and IV, and found that radical surgical efforts are connected with improvement of prognosis. They considered that the borders of the pelvic disease could be determined with difficulty in cases of extensive involvement of the 


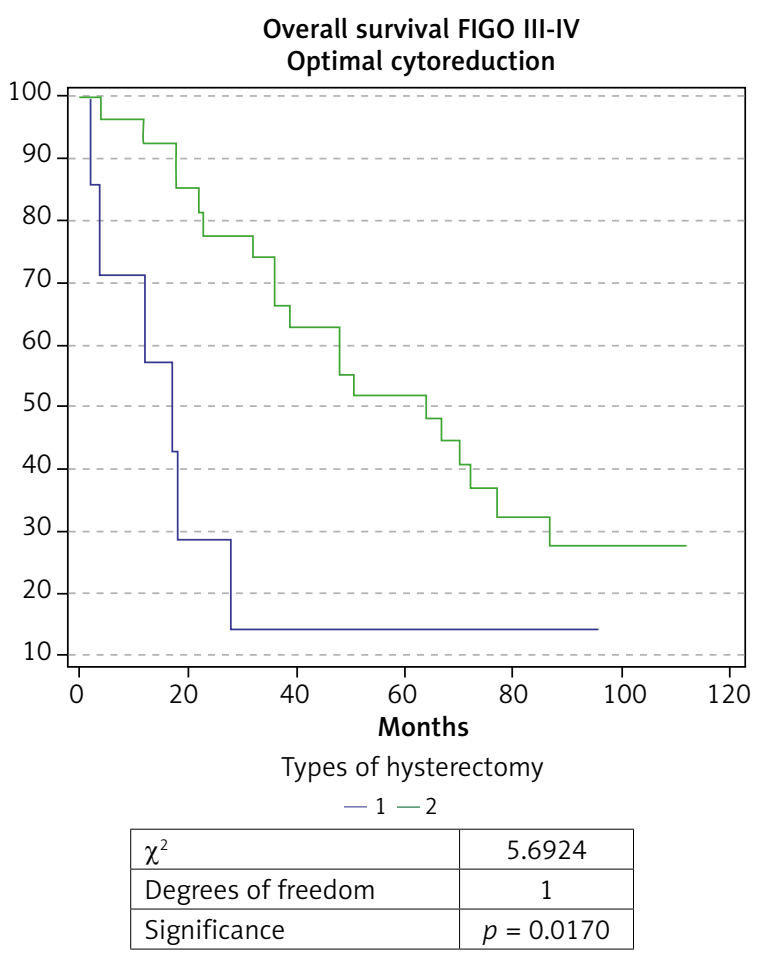

Fig. 5. Kaplan-Meier curve for overall survival rate of patients with ovarian carcinoma of FIGO stages III-IV, and achieved optimal cytoreduction, divided into two groups according to type of hysterectomy: group 1 with total abdominal hysterectomy (TAH), and group 2 with extended hysterectomy (EH)

pelvis, and there is a possibility for the residual tumor to be omitted. In their cohort of patients, they studied a subgroup with optimal cytoreduction, in which they found a statistically significant benefit of radical pelvic surgery for survival, underlining that the patients benefited from it in the cases of possible extirpation of the disease in the abdominal region as well [7]. The analysis of our data also demonstrated that the attempt for radicality in the pelvic region through $\mathrm{EH}$ with a retroperitoneal approach, with or without pelvic peritonectomy, or rectosigmoid resection brought about a significant benefit for the survival rate of patients with residual tumor of $0-1 \mathrm{~cm}$.

The indications for performing of $\mathrm{EH}$ were summarized by Eisenkop in 1991 [8]. The two main indications are extensive confluent tumor involvement of the pelvis and rectouterine pouch, and the subjective surgeons' assessment of the possibility of the complete removal of the disease using non-radical procedures. The clinical benefit of the $\mathrm{EH}$ is based on its demonstrated efficacy for the removal of the whole pelvic disease and its contribution for reduction of the size of residual tumor [9].

Once an optimal cytoreduction is achieved (residual tumor of 0-1 cm), the EH gives an advantage over the TAH in terms of survival. A probable reason for that is the greater radicality of the procedure with removal of

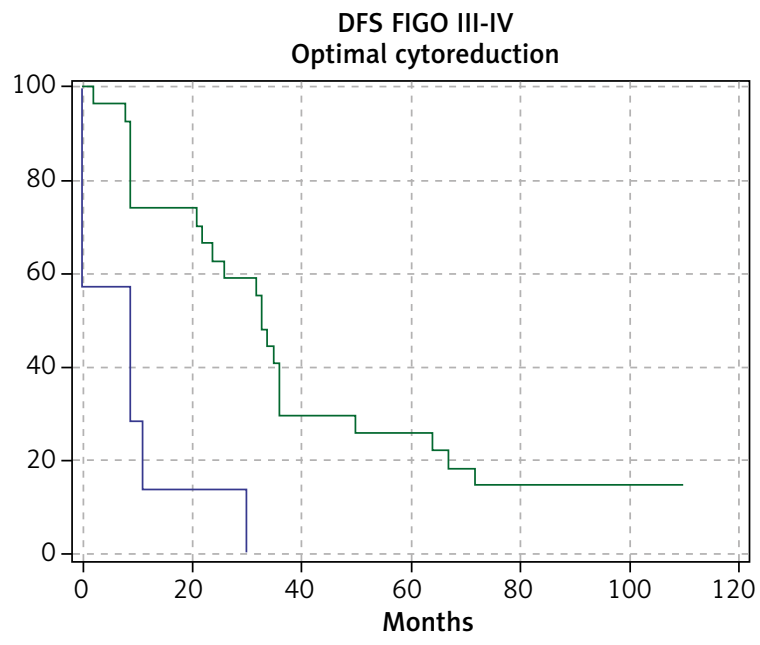

Types of hysterectomy

$-1-2$

\begin{tabular}{|l|c|}
\hline$\chi^{2}$ & 13.7420 \\
\hline Degrees of freedom & 1 \\
\hline Significance & $p=0.0002$ \\
\hline
\end{tabular}

Fig. 6. Kaplan-Meier curve for disease-free survival (DFS) rate of patients with ovarian carcinoma of FIGO stages III-IV and achieved optimal cytoreduction, divided into two groups according to type of hysterectomy: group 1 with total abdominal hysterectomy (TAH), and group 2 with extended hysterectomy (EH)

macroscopically invisible (microscopic) tumor lesions, as it reduces the residual tumor burden and supports the subsequent chemotherapy. Furthermore, Pereira et al. - as well as our study - showed that it also removed an „invisible” residual tumor such as parametrial and vaginal invasion, when the tumor developed by direct extension [10]. These facts underlined the significance of the retroperitoneal approach and $\mathrm{EH}$ for the cytoreductive treatment and identification of metastatic disease.

The clinical benefit of the $\mathrm{EH}$ is based on its demonstrated efficacy for removal of the whole pelvic disease, and its contribution to the size of residual tumor [11]. Optimal cytoreduction is achievable in $74 \%$ to $100 \%$ of cases of $\mathrm{EH}$, with almost complete clearance of the advanced pelvic tumor [7, 8, 11-17].

It appears that the successes of the EH for the optimal cytoreduction may not influence the clinical outcome in all patients with AOC, but it may be beneficial only where attempts for resection of disease in the upper abdomen are undertaken [6, 7]. Amongst our patients with an overall survival rate of up to one year after the operation, we found a significant percentage $(67 \%)$ of high tumor dissemination (in 14 out of 21 patients), i.e., residual tumor in the upper abdomen (liver, diaphragm) despite the $\mathrm{EH}$. 
This study has some limitations and strengths. The limitations are the small number of patients and heterogeneous types of ovarian cancer. The strengths are that we present the 5-year survival rate and that one surgical team performed all procedures (the procedure is standardized). We are continuing with this study and with follow-up of the included patients and we will be ready with the 10-year survival rate soon.

\section{Conclusions}

We are confident of the $\mathrm{EH}$ with a retroperitoneal approach being a surgical technique which allows complete extirpation of the pelvic tumor and leads to improvement of the survival rate as a result of removal of macroscopically invisible lesions as well.

\section{Disclosure}

The authors report no conflict of interest.

\section{References}

1. De Angelis R, Sant M, Coleman MP, et al. Cancer Survival in Europe 19992007 by Country and Age: Results of EUROCARE-5 - a Population-Based Study. Lancet Oncol 2014; 15: 23-34.

2. Matz M, Coleman MP, Carreira H, et al. Worldwide Comparison of Ovarian Cancer Survival: Histological Group and Stage at Diagnosis (CONCORD-2) Gynecol Oncol 2017; 144: 396-404.

3. NCCN Guideline Ovarian Cancer Version 1.2019. Available at: https://www. nccn.org/professionals/physician_gls/pdf/ovarian.pdf (access: 20 January 2020).

4. Hudson CN, Chir M. A Radical Operation for Fixed Ovarian Tumors. J Obstet Gynaecol Br Commonw 1968; 75: 1155-1156.

5. Prat J. FIGO Committee on Gynecologic Oncology. Staging Classification for Cancer of the Ovary, Fallopian Tube, and Peritoneum. Int J Gynaecol Obstet 2014; 124: 1-5.

6. Benedetti-Panici P, Maneschi F, Scambia G, et al. The Pelvic Retroperitoneal Approach in the Treatment of Advanced Ovarian Carcinoma. Obstet Gynecol 1996; 87: 532-538.

7. Aletti GD, Dowdy SC, Gostout BS, et al. Aggressive Surgical Effort and Improved Survival in Advanced-Stage Ovarian Cancer. Obstet Gynecol 2006; 107: 77-85.

8. Eisenkop SM, Nalick RH, Teng NN. Modified Posterior Exenteration for Ovarian Cancer. Obstet Gynecol 1991; 78: 879-885.

9. Bristow RE, Karlan BY, Chi DS. Surgery for Ovarian Cancer, 2nd ed. Informa Healthcare 2010.

10. Pereira A, Pérez-Medina T, Magrina JF, et al. The Impact of Pelvic Retroperitoneal Invasion and Distant Nodal Metastases in Epithelial Ovarian Cancer. Surg Oncol 2014; 23: 40-44.

11. Clayton RD, Obermair A, Hammond IG, et al. The Western Australian Experience of the Use of En Bloc Resection of Ovarian Cancer with Concomitant Rectosigmoid Colectomy. Gynecol Oncol 2002; 84: 53-57.

12. Barnes W, Johnson J, Waggoner S, et al. Reverse Hysterocolposigmoidectomy (RHCS) for Resection of Panpelvic Tumors. Gynecol Oncol 1991; 42: 151-155.

13. Bristow RE, del Carmen MG, Kaufman HS, Montz FJ. Radical Oophorectomy with Primary Stapled Colorectal Anastomosis for Resection of Locally Advanced Epithelial Ovarian Cancer. J Am Coll Surg 2003; 197: 565-574.

14. Hertel H, Diebolder H, Herrmann J, et al. Is the Decision for Colorectal Resection Justified by Histopathologic Findings: a Prospective Study of 100 Patients with Advanced Ovarian Cancer. Gynecol Oncol 2001; 83: 481-484.
15. Obermair A, Hagenauer S, Tamandl D, et al. Safety and Efficacy of Low Anterior En Bloc Resection as Part of Cytoreductive Surgery for Patients with Ovarian Cancer. Gynecol Oncol 2001; 83: 115-120.

16. Sainz de la Cuesta R, Goodman A, Halverson SS. En Bloc Pelvic Peritoneal Resection of the Intraperitoneal Pelvic Viscera in Patients with Advanced Epithelial Ovarian Cancer. Cancer J Sci Am 1996; 2: 152-157.

17. Sugarbaker PH. Complete Parietal and Visceral Peritonectomy of the Pelvis for Advanced Primary and Recurrent Ovarian Cancer. Cancer Treat Res 1996; 81: 75-87. 